\title{
Network target for screening synergistic drug combinations with application to traditional Chinese medicine
}

\author{
Shao Li*, Bo Zhang, Ningbo Zhang \\ From The 4th International Conference on Computational Systems Biology (ISB 2010) \\ Suzhou, P. R. China. 9-11 September 2010
}

\begin{abstract}
Background: Multicomponent therapeutics offer bright prospects for the control of complex diseases in a synergistic manner. However, finding ways to screen the synergistic combinations from numerous pharmacological agents is still an ongoing challenge.

Results: In this work, we proposed for the first time a "network target"-based paradigm instead of the traditional "single target"-based paradigm for virtual screening and established an algorithm termed NIMS (Network targetbased Identification of Multicomponent Synergy) to prioritize synergistic agent combinations in a high throughput way. NIMS treats a disease-specific biological network as a therapeutic target and assumes that the relationship among agents can be transferred to network interactions among the molecular level entities (targets or responsive gene products) of agents. Then, two parameters in NIMS, Topology Score and Agent Score, are created to evaluate the synergistic relationship between each given agent combinations. Taking the empirical multicomponent system traditional Chinese medicine (TCM) as an illustrative case, we applied NIMS to prioritize synergistic agent pairs from 63 agents on a pathological process instanced by angiogenesis. The NIMS outputs can not only recover five known synergistic agent pairs, but also obtain experimental verification for synergistic candidates combined with, for example, a herbal ingredient Sinomenine, which outperforms the meet/min method. The robustness of NIMS was also showed regarding the background networks, agent genes and topological parameters, respectively. Finally, we characterized the potential mechanisms of multicomponent synergy from a network target perspective.

Conclusions: NIMS is a first-step computational approach towards identification of synergistic drug combinations at the molecular level. The network target-based approaches may adjust current virtual screen mode and provide a systematic paradigm for facilitating the development of multicomponent therapeutics as well as the modernization of TCM.
\end{abstract}

\section{Background}

Multicomponent therapeutics, in which two or more agents interact with multiple targets simultaneously, is considered as a rational and efficient form of therapy designed to control complex diseases [1,2]. Here "agent" refers to medicinal entities, chemical substances, herbs and the like with pharmacological or biological activities.

\footnotetext{
* Correspondence: shaoli@mail.tsinghua.edu.cn

MOE Key Laboratory of Bioinformatics and Bioinformatics Division, TNLIST / Department of Automation, Tsinghua University, Beijing 100084, China Full list of author information is available at the end of the article
}

One of the fundamental advantages of multicomponent therapeutics is the production of "synergy", that is, the combinational effect to be greater than the sum of the individual effects, making multicomponent therapeutics a systematic approach, rather than the reductionism of an additive effect. Understanding multicomponent synergy is critical for developing a novel strategy to conquer complex diseases. It is believed that combinations of agents can effectively reduce side effects and improve adaptive resistance, thereby increasing the likelihood of
C Biomed Central 
conquering complex diseases, such as cancer, in a synergistic manner [3].

Evaluation of multicomponent synergy is usually implemented experimentally in a case-by-case approach [4] and evaluated using the reference models of additivism to recognize synergy such as the Bliss independence model [5], the Loewe additivism model [6] and the Combination Index theorem [7]. However, large number of possible agent combinations will be formed even in the case of a small collection of therapeutic agents. Therefore, although some experimental methods have been launched to screen favourable drug combinations by disease-relevant phenotypic assays [8], the highthroughput identification of synergistic agent combinations arising from numerous agents remains an unresolved issue [9]. By way of contrast, computational approaches that take advantage of the rapid accumulation of massive data may provide a more promising and desirable method for multicomponent drug studies. Currently, computational efforts for the evaluation of multicomponent therapeutics mainly focus on two directions. The first direction is to identify and optimize multiple target interventions by modelling signaling pathways or specific processes and is usually applied to small scale problems $[10,11]$. One of limitations of this approach is the fact that crosstalks, feedbacks or interactions among pathways are widely present in complex diseases, suggesting that pathways should be integrated rather than treated separately $[12,13]$. The second newly developing direction is to measure the efficacy of drugs, especially multi-target drugs, by using network biology approaches [14]. However, the realistic method remains to be established and the association between drug actions and network properties is not precisely known. Thus, finding ways to evaluate multicomponent therapeutics and sort order for synergistic agent combinations is still a considerable challenge. Novel computational approaches are urgently required for feasible and efficient identification of multicomponent synergy.

Recently, computational systems biology approaches as well as our previous studies have been enhancing our understanding of various aspects of complex diseases, including the identification of disease-related genes or functional modules, and the recognition of redundant, adaptable and system mechanisms in diseases [15-17]. Now, we are standing at the portal of a new era to bridge molecular states to physiological states as well as various disease states through the biological networks that sense genetic and environmental perturbations [18]. To keep in line with new developments, researchers have also started to change their way of thinking in terms of drug-treated complex biological systems, and studies such as network pharmacology [19] have been springing up. Against this background, we propose a novel concept, "network target", with the attempt to update current single target-based or multiple targetbased drug studies. We roughly defined the "network target" as a therapeutic target that is derived from systematic interventions of the biological network (including the network state and its pivotal elements) underlying a disease or pathological process. The concept of network target considers simultaneously the disease mechanisms and drug actions on a network basis, and a network target for a certain disease may correspond to a variety of single-component or multicomponent therapeutics.

On the other hand, while the scientific community has high expectations for the coming network pharmacology [19], this new field should be composed of two main approaches due to our poor understanding of cell behaviours and drug-protein interactions: 1) Bottom-up: Addition of well-known molecular drugs and observation of synergistic effects; 2) Top-down: Reduction of more general formulae to its minimal elements that keep its beneficial properties. In this regard, an empirical system of multicomponent therapeutics, traditional Chinese medicine (TCM), may have the potential of addressing a relationship between multicomponents and drug synergistic effects. Having been evolved over 3,000 years, TCM is characterized by the use of Herbal Formulae (Fu-Fang) that are usually grouped by two or more medicinal herbs and capable of systematically controlling various diseases such as angiogenic disorders [20] via potentially synergistic herb interactions [21,22]. For instance, the Realgar-Indigo naturalis Formula has an effect on promyelocytic leukemia via the action mechanism of synergy among its components [23]. Thus, the multicomponent synergy in Chinese herbs is of great significance for understanding TCM and for new drug discovery. Although this is still an open question, it is believed that the rich body of TCM experience in combined use of herbs may provide an excellent model for studying synergistic effects among different components [24], and the systems biology approaches could shed light on the mystery of TCM [22,25].

In this work, we report a novel method, called NIMS (Network target-based Identification of Multicomponent Synergy), to address the network target-based virtual screen and assess the synergistic strength of multicomponent therapeutics. NIMS measures synergistic agent combinations by creating and integrating two parameters, namely Topology Score and Agent Score. Next, NIMS was applied to prioritize synergistic combinations from 63 agents including 61 herbs or herb compounds as well as five agent pairs with known synergistic effects containing 2 additional chemicals 5-fluorouracil and Rapamycin. One of NIMS outputs was then subjected to experimental verification. We hope the network target- 
based approaches will improve our understanding of multicomponent therapeutics in terms of complex biological systems.

\section{Results}

\section{Pipeline of NIMS}

The rationale of the network target concept and NIMS is to transfer the relationship among agents to the interactions among the targets or responsive gene products of agents in the context of a biological network specific for a disease or pathological process. This hypothesis may be reasonable in many situations especially when synergy occurs only if the effects of individual agents are mediated through independent action mechanisms. In NIMS, a set of genes or gene products affected by an agent are termed agent genes, and the disease-specific biological network serves as the background network to perform NIMS. Then, two elements in NIMS, Topology Score (TS) and Agent Score $(A S)$, are proposed to evaluate agent interactions.

As shown in Figure 1, TS is derived from topological features of the background network related to certain disease conditions and drug actions. From the network target perspective, the achilles' heel of the biological network underlying a certain disease is more likely to become the attack points of drugs. Thus, we assume that the more important the agent gene as a network node is, the stronger effect on the disease the agent will produce. To determine the importance of an agent gene as a node in the network, we propose a node importance score, (IP $(v)$, here $v$ denotes a vertex / node), by integrating degree [26], betweenness [27] and closeness [28], three network centrality indexes that have been used to define the network properties of drug targets separately or collectively [29]. Moreover, we suppose that if an agent pair produces synergy, their agent genes should be adjacent in the network. Accordingly, for a candidate agent pair agent ${ }_{1}$ and agent $_{2}$, we defined a topology-dependent score, TS, to evaluate both the importance score $(I P(v))$ of agent $_{1}$ genes and agent $_{2}$ genes and the network distance between these two gene sets. $T S_{1,2}$ is given by:

$$
T S_{1,2}=\frac{1}{2} \times\left(\frac{\sum_{i} I P_{1}(i) \times \exp \left(-\min \left(d_{i, j}\right)\right)}{\sum_{i} I P_{1}(i)}+\frac{\sum_{j} I P_{2}(j) \times \exp \left(-\min \left(d_{j, i}\right)\right)}{\sum_{j} I P_{2}(j)}\right)
$$

where $I P_{1}(i)$ for agent $t_{1}$ genes and $I P_{2}(j)$ for agent 2 genes are calculated by integrating Betweenness, Closeness and a variant of the Eigenvector PageRank [30] through Principal Component Analysis (PCA). The negative exponential function is utilized to weigh the interaction of two agents based on the shortest path length. The $\min \left(d_{i, j}\right)$ is the minimum shortest path from

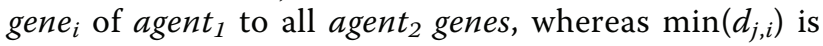
the minimum shortest path from gene ${ }_{j}$ of agent $t_{2}$ to all agent $_{1}$ genes. We only consider the nearest connection between agent ${ }_{1}$ genes and agent ${ }_{2}$ genes in the network. The two terms in the brackets are dual and represent the synergy strength measurement for a combination of agent $_{1}$ and agent . $_{2}$

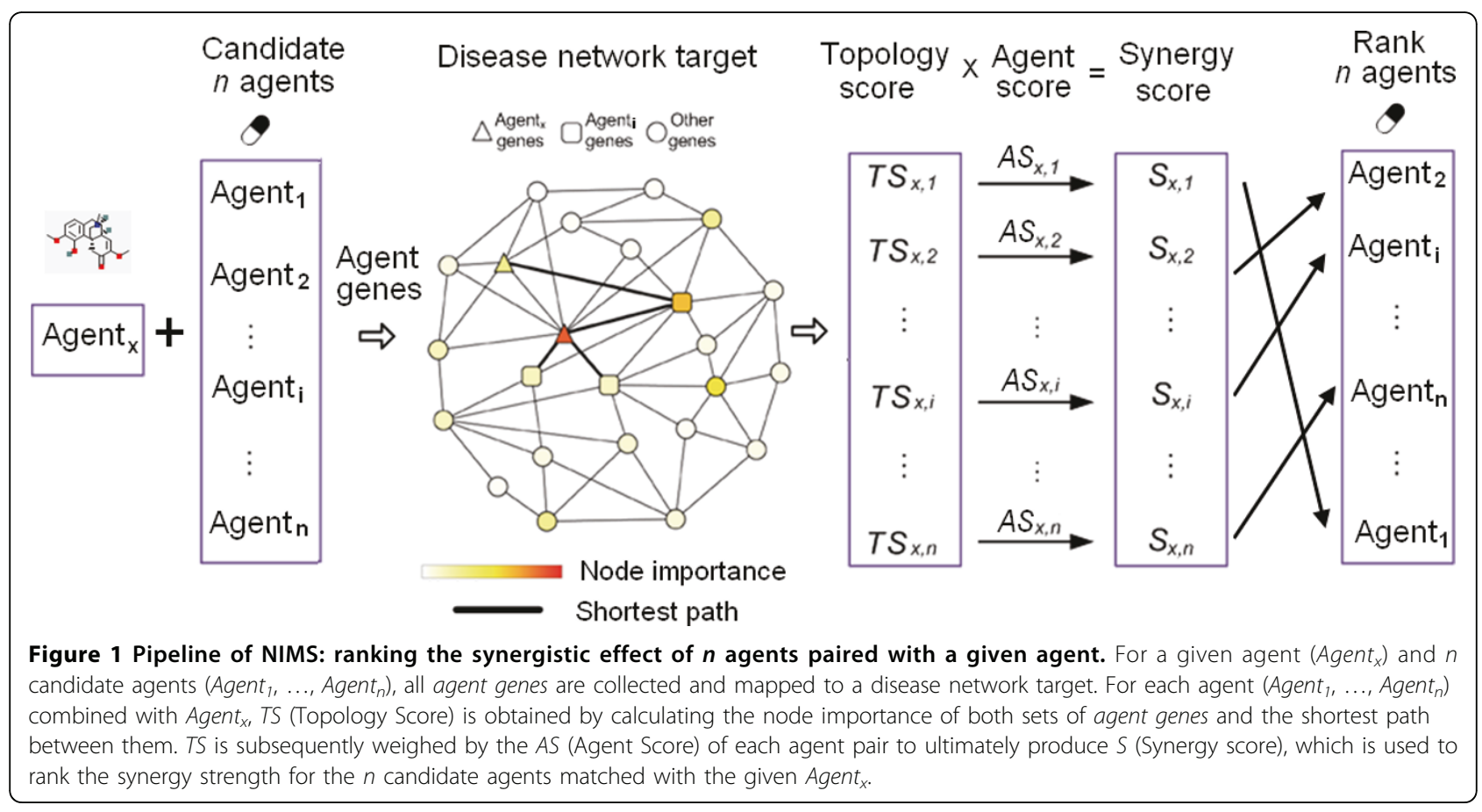


As agents with independent action mechanisms but treating similar diseases may be more likely to produce synergistic effect, we also introduced $A S$, a concept transferred from the disease phenotype similarity [31], to quantify the similarity score of two agents and finetune the TS results. Here, if an agent gene falls into the gene set of a phenotype recorded in the OMIM (Online Mendelian Inheritance in Man) database, this phenotype will be identified as an agent phenotype for the given agent. The similarity between two agent phenotypes quantifies the overlap of their OMIM descriptions and is calculated by a text mining method [31] (See Methods). The $A S$ for agent ${ }_{1}$ and agent $_{2}$ is given by $A S_{1,2}=\frac{\sum_{i, j} P_{i, j}}{N}$, where $P_{i, j}$ is the similarity score between phenotype $_{i}$ of agent $_{1}$ and phenotype $e_{j}$ of agent $t_{2}$, and $N$ is the total number of phenotype pairs.

Ultimately, NIMS produces the synergy score, $S_{1,2}$, for agent $_{1}$ and agent $_{2}$ by calculating $S_{1,2}=T S_{1,2} \times A S_{1,2}$, which denotes the node importance, network adjacency and action similarity of two gene sets of $a g e n t_{1}$ and agent $_{2}$. A high score means a great probability of synergy. Note that currently NIMS only measures the synergy of combinational agents with independent mechanisms according to the Bliss independent theory [5], so we roughly set the valid range of the NIMS score from 0 to 0.9 . When the score is larger than 0.9 , the two agents in combination are more likely to act on the same gene sets and in contradiction with the independence assumption. For these agent combinations, we may need more information to distinguish their interaction modes.

\section{Application and experimental verification of NIMS}

We applied NIMS to prioritize synergistic agent pairs from 63 manually collected agents (See Methods) and estimated their effects on angiogenesis, a key pathological process in various diseases such as cancer and rheumatoid arthritis [32], with the network constructed by our LMMA approach previously [17]. The NIMS synergy scores for all agent pairs against the angiogenesis network ranged from 0.199270 to 0.012959 , with TS score from 0.814868 to 0.103790 and $A S$ score from 0.262459 to 0.107882 , respectively. From the outputs of NIMS, we firstly checked the rank of five agent pairs with known synergy in every 62 pairs for a given agent. As shown in Table 1, the synergy scores of both 5-fluorouracil (5-FU) combined with Vinblastine [33] and 5-FU combined with Rapamycin [34] entered the top three. Three other synergistic pairs, Vinblastine and Camptothecin [35], Genistein and Camptothecin [36], and Genistein and Rapamycin [37], also earned high marks and ranked in the top layer. We then used, respectively, three global background networks including the global protein-protein interaction (PPI) network and two kinds of global pathway networks (Keep Node Content and Merge Node Content, KNC and MNC) (See Methods) to calculate the synergy score. Results showed that NIMS is relatively robust to different background networks in these cases (Table 1).

Next, an in vitro assay was conducted to validate NIMS predictions. Sinomenine, an anti-angiogenic alkaloid that extracted from a TCM commonly used herb named Sinomenium acutum [20,38], was selected as the seed agent (as Agent $t_{x}$ in Figure 1). Agent combinations were sampled from five intervals of the rank list composed of all 62 agents matched with Sinomenine. Here, we only considered commercially available agents with known chemical structures. This restriction left five Sinomenine partners, namely Luteolin, Quercetin, Honokiol, Matrine and Paeoniflorin. To determine the synergy strength of the agent pairs, low-dose combinations with more than a

Table 1 NIMS ranks against four types of background networks

\begin{tabular}{|c|c|c|c|c|c|}
\hline \multirow[b]{2}{*}{ Given agent } & \multirow[b]{2}{*}{ Partner agent } & \multicolumn{4}{|c|}{ Rank among 62 agent pairs \# } \\
\hline & & Angiogenesis network (NIMS score) & PPI & KNC & MNC \\
\hline \multirow[t]{2}{*}{5 -fluorouracil } & Vinblastine* & $2(0.18104)$ & 1 & 2 & 2 \\
\hline & Rapamycin* & $3(0.13744)$ & 2 & 3 & 26 \\
\hline Vinblastine & Camptothecin* & $1(0.19927)$ & 1 & 1 & 3 \\
\hline \multirow[t]{2}{*}{ Genistein } & Camptothecin* & $2(0.12070)$ & 3 & 2 & 2 \\
\hline & Rapamycin* & $6(0.11533)$ & 4 & 7 & 4 \\
\hline \multirow[t]{5}{*}{ Sinomenine } & Matrine & $4(0.10923)$ & 6 & 3 & 11 \\
\hline & Honokiol & $8(0.10142)$ & 5 & 9 & 16 \\
\hline & Luteolin & $10(0.10007)$ & 11 & 17 & 6 \\
\hline & Quercetin & $14(0.09835)$ & 20 & 5 & 3 \\
\hline & Paeoniflorin & $29(0.08215)$ & 26 & 29 & 31 \\
\hline
\end{tabular}

*: Agent pairs with known synergistic effects.

\#: For each given agent, there are totally 62 candidate agent pairs. PPI, protein-protein interaction network. KNC, Keep Node Content pathway network. MNC, Merge Node Content pathway network. 
$70 \%$ inhibition rate were regarded as effective [39]. Using the Maximum Increased Inhibition Rate (MIIR) measure for each combination (Figure 2), we found that the highest MIIR 26.83\% was reached by Sinomenine combined with Matrine $((\mathrm{S}):(\mathrm{M}))$, whereas the lowest MIIR $1.86 \%$ was reached by Sinomenine combined with Paeoniflorin $((\mathrm{S}):(\mathrm{P}))$. This rank order of agent pairs is identical to the order predicted by NIMS when against the angiogenesis network, and such a performance is superior to those against three global networks (Table 1).

\section{Robustness of NIMS}

NIMS integrated three measures, namely Betweenness, Closeness and PageRank to capture the node importance $I P(v)$ from different aspects. In the undirected angiogenesis network, we found that all three measures are highly

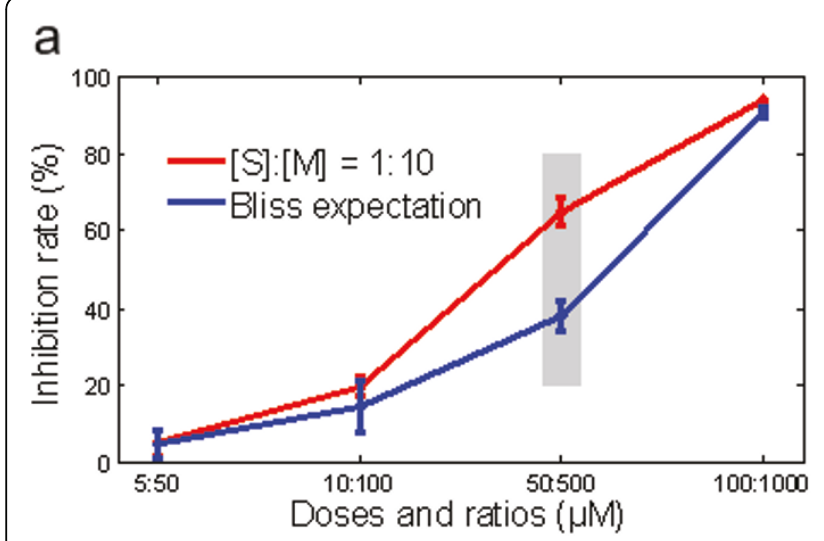

C
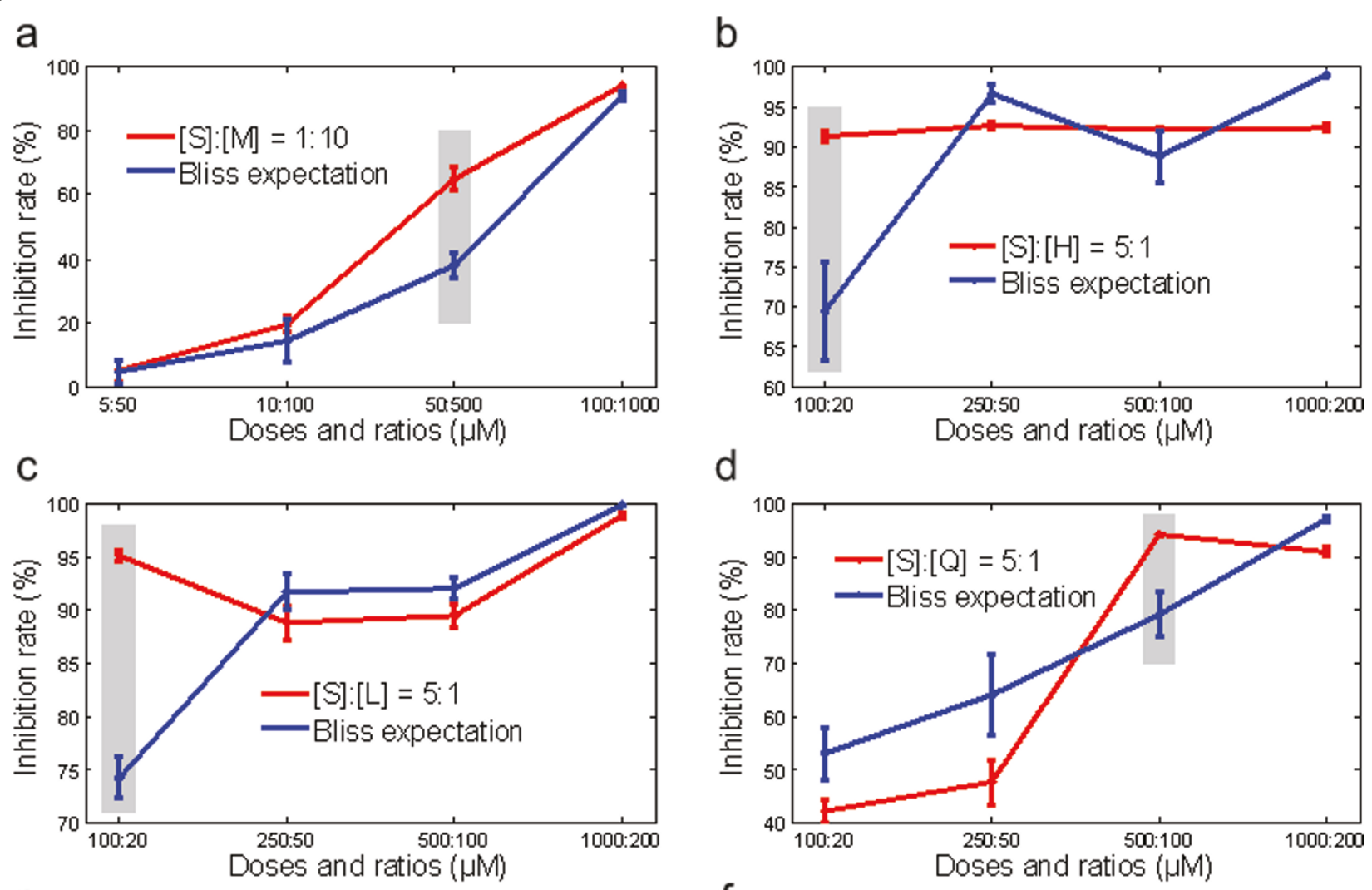

d
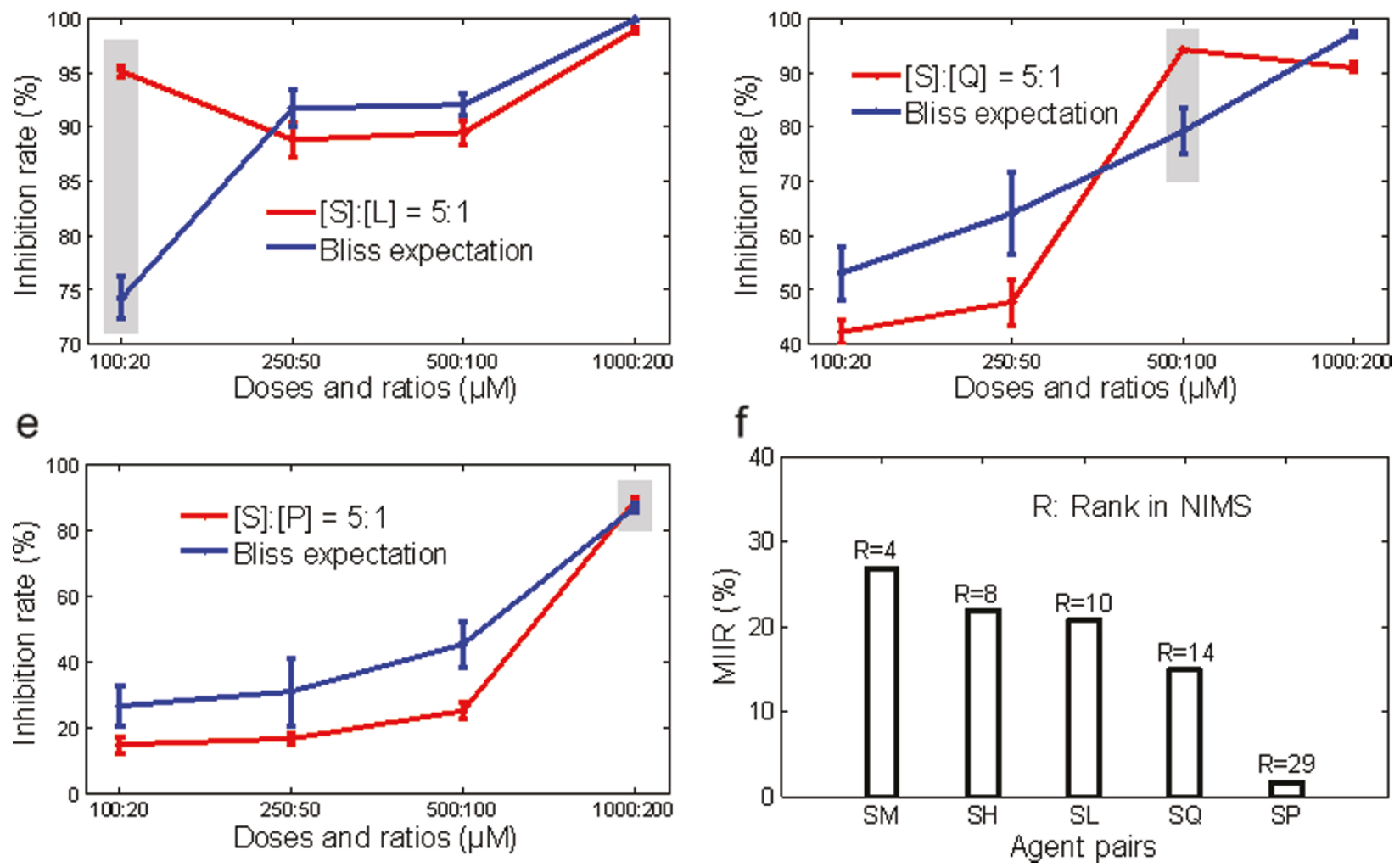

Figure 2 Anti-angiogenesis synergistic effects of five agent pairs. a-e. The red line denotes the inhibition rate of Human Umbilical Vein Endothelial Cells (HUVEC) proliferation in a dose-dependent manner. The blue line denotes the additive effects calculated by the Bliss independence model. The gray column denotes the optimal dose and ratio of each pair. $\mathbf{f}$. The value of the maximum increased inhibition rate (MIIR) for the synergistic effects produced by five agent pairs corresponds well with the NIMS ranks against the angiogenesis network. The proportion of two agents is determined by following the same ratio of the two agent's IC50 values. 
correlated and the majority (94.81\%) of their variance can be explained by the primary eigenvector. However, these three centrality measures could not replace one another, especially in the directed networks. Thus, we integrated these three centrality measures to address the node importance from different angles. Furthermore, the positive role of $A S$ in NIMS was also shown in the agent pair rankings. In the case study, the $A S$ scores of Matrine, Honokiol, Luteolin, Quercetin and Paeoniflorin separately combined with Sinomenine were 0.1708, 0.1590, $0.1705,0.1611$ and 0.1414 , respectively. These scores reached an approximate rank with that resulted from network topologies alone. The removal of the AS scores ranked Luteolin ahead of Quercetin, suggesting that the integration of $A S$, which reflects current knowledge about complex diseases and agent actions, could improve the predictive accuracy of NIMS by weighing TS.

The robustness of NIMS was also addressed with respect to both agent genes and the background network. By adding or removing agent genes randomly, the permutation test results showed that the Spearman Rank Correlation Coefficient (SRCC) was relatively stable when adding genes, but the SRCC decreased dramatically when some key genes were removed (Figure $3 \mathrm{a}$ and Figure $3 \mathrm{~b}$ ). The results evidence that the NIMS synergy score may be determined largely by some key agent genes, and the rank results will remain stable as long as these key genes are retained. Such phenomena also agree well with that the power law networks are robust with respect to deletion of random nodes, but fragile with respect to deletion of hubs [40]. Moreover, by deleting or importing additional interactions at different percentages in the angiogenesis network, we found that the NIMS outputs were quite stable even when $50 \%$ of the edges were randomly removed or added (Figure 3c), indicating that NIMS is insensitive to both incompleteness and noise regarding the background network.

\section{Comparison with meet/min}

To determine whether the synergy rank of agent pairs could be obtained from corresponding agent genes alone, regardless of network knowledge, we used the meet/min method, a similarity measurement between two gene sets that discards the network information [41], to rank the agent pairs. The meet/min method is believed to be simple but effective and non-biased [41]. Because the NIMS score and the meet/min coefficient (ranging from 0 to 1 ) will both reach their maximum when the gene set of one agent is merely the subset of that of the other agent, we only investigated agent combinations with valid scores from 0 to 0.9 . In general, a relatively high correlation $(\mathrm{SRCC}=0.6251)$ between the meet/min coefficient and the NIMS synergy score was observed for all agent pairs. However, compared with the experimental results, the performance of the meet/ min method was relatively poor in ranking synergistic pairs with Sinomenine (Table 2).

\section{NIMS synergy and GO function}

We measured Gene Ontology (GO) co-annotations to advance understanding of the underlying synergy mechanism for agent pairs predicted by NIMS. All three GO categories, Biological Processes, Cellular Components and Molecular Functions, were considered. As shown in Table 3, weak correlations were observed between the NIMS synergy scores and the GO similarity scores calculated from genes of each agent pairs. Results showed that agents with synergy may not target the same functional processes.

\section{Features of synergistic agent combinations on the angiogenesis network target}

Practically, we treat the angiogenesis network target as core subnetworks of angiogenesis network which contains the intersection of a set of shortest path subnetworks
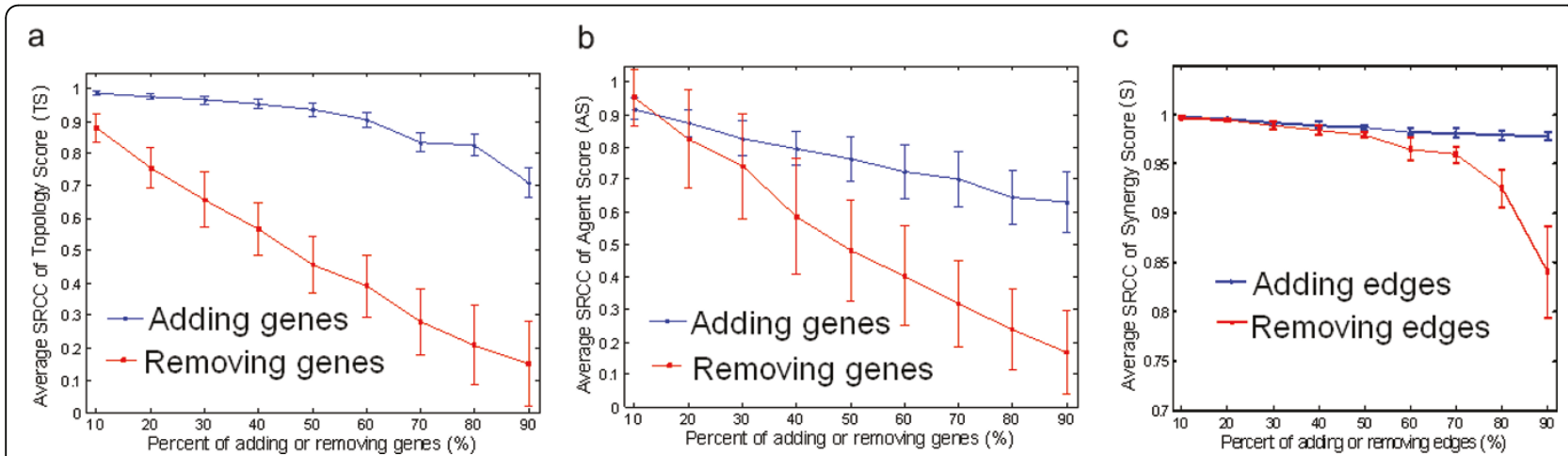

Figure 3 Permutation tests to assess the robust performance of NIMS. The permutations are performed by evaluating fluctuations of (a) TS (Topology Score), (b) AS (Agent Score), and (c) the background network (angiogenesis network) and calculated by the average SRCC (Spearman rank correlation coefficient) between the permutation outputs and the original scores. 
Table 2 Synergy ranks of five Sinomenine pairs resulted from NIMS, meet/min and cell experiment

\begin{tabular}{|c|c|c|c|c|c|}
\hline \multirow[t]{2}{*}{ Agent matched with Sinomenine } & \multicolumn{3}{|c|}{ NIMS rank } & \multirow[t]{2}{*}{ The meet/min rank } & \multirow[t]{2}{*}{ Experimental rank } \\
\hline & $A S \times T S$ & TS & AS & & \\
\hline Matrine & 4 & 5 & 10 & 20 & 1 \\
\hline Honokiol & 8 & 6 & 32 & 19 & 2 \\
\hline Luteolin & 10 & 21 & 12 & 21 & 3 \\
\hline Quercetin & 14 & 14 & 26 & 29 & 4 \\
\hline Paeoniflorin & 29 & 22 & 52 & 6 & 5 \\
\hline
\end{tabular}

AS, Agent Score; TS, Topology Score.

associated with individual or combinational drug actions. To learn the exact features on the angiogenesis network target derived from agent combinations with different NIMS scores, we mapped the responsive genes of 5-flourourcil, Vinblastine, Sinomenine, Matrine and Paeoniflorin to the network target and the detailed network features especially pathway crosstalks and feedback loops were analyzed. As shown in Figure 4, we found that the network target could capture different synergistic responses induced by three agent combinations with different NIMS synery scores. For example, 5-flourourcil and Vinblastine can affect KDR protein complex, the crosstalk between AKT1 and MAPK1 pathways, the PTEN feedback loop as well as two biological processes, endothelial cell proliferation and apoptosis, and four hub nodes (KDR, MAPK1, JUN and TP53). The network target affected by Sinomenine and Matrine contains the crosstalk with EGFR, KDR and TNFRSF1A pathways, the PTEN feedback loop, as well as, four biological processes closely associated with angiogenesis and two hub nodes (JUN and TP53). However, Sinomenine and Paeoniflorin with lower synergy score can only affect two biological processes and one hub node (TP53) (Figure 4).

\section{Characterizing the mechanisms of multicomponent synergy from a network target perspective}

Despite the widespread occurrence of multicomponent therapeutics, the molecular mechanisms that underlie drug synergy remain unclear. Based on the above computational and experimental results of NIMS, we demonstrate that the network target can nicely interpret the multicomponent synergy by its latent network

Table 3 Correlation of the NIMS synergy score with agent genes' GO co-annotations

\begin{tabular}{cccc}
\hline \multirow{2}{*}{ Categories } & \multicolumn{3}{c}{$\begin{array}{c}\text { Correlation of the NIMS score with GO similarity } \\
\text { score }\end{array}$} \\
\cline { 2 - 4 } & $\begin{array}{c}\text { Biological } \\
\text { Processes }\end{array}$ & $\begin{array}{c}\text { Cellular } \\
\text { Components }\end{array}$ & $\begin{array}{c}\text { Molecular } \\
\text { Functions }\end{array}$ \\
\hline SRCC & 0.1649 & 0.0641 & 0.1571 \\
\hline $\begin{array}{c}\text { p-value of } \\
\text { SRCC }\end{array}$ & 0.1963 & 0.617 & 0.2182 \\
\hline
\end{tabular}

GO, Gene Ontology; SRCC, Spearman Rank Correlation Coefficient. topology properties. We hence give a generalization of the network target concept and NIMS parameters to formalize our viewpoints on drug synergistic mechanisms. As shown in Figure 5, the shortest path distance $\left(\min \left(d_{i, j}\right)\right.$ in NIMS) can describe the protein complexes, crosstalks as well as feedback loops in the network formed by genes associated with two agents (Figure 5a), the hub and betweenness (IP $(v)$ in NIMS) denotes the importance of genes or stimuli-influenced number of molecules two agents affected (Figure 5b), and functional modules means the biological processes two agents targeted (Figure 5c). It is important to note that these findings match well with the synergy phenomena present in complex biological systems. The available evidences showed that molecular synergisms can be emerged from different aspects, for example, protein complexes in cell-regulatory systems [42], crosstalk [43-47] and feedback control in the structures of signal pathways $[48,49]$, stimuli-influenced number of molecules (e.g. number of activated enzymes, receptors, channels or transcription factors) [50,51] and gene expression profile [52] in signal transduction process. Thus, from the network target perspective, we can gain a comprehensive understanding of drug synergistic mechanisms on the basis of complex biological systems.

\section{Discussion}

Recently, with the growing understanding of complex diseases, the focus of drug discovery has shifted from the well-accepted "one target, one drug" model designed toward a single target to a new "multi-target, multidrug" model aimed at systemically modulating multiple targets $[19,53]$. In this work, we proposed the concept of "network target", which treats the disease-specific biological network and its key elements as a therapeutic target, and established a NIMS approach to prioritize the multicomponent synergy. NIMS combines network topology and agent similarity, with regard to agent genes as well as phenotypes. To demonstrate the capability of NIMS, we applied this algorithm to the prioritization of synergistic anti-angiogenesis agent pairs from an empirical multicomponent therapeutic system, TCM. Our results show that NIMS, especially when used 


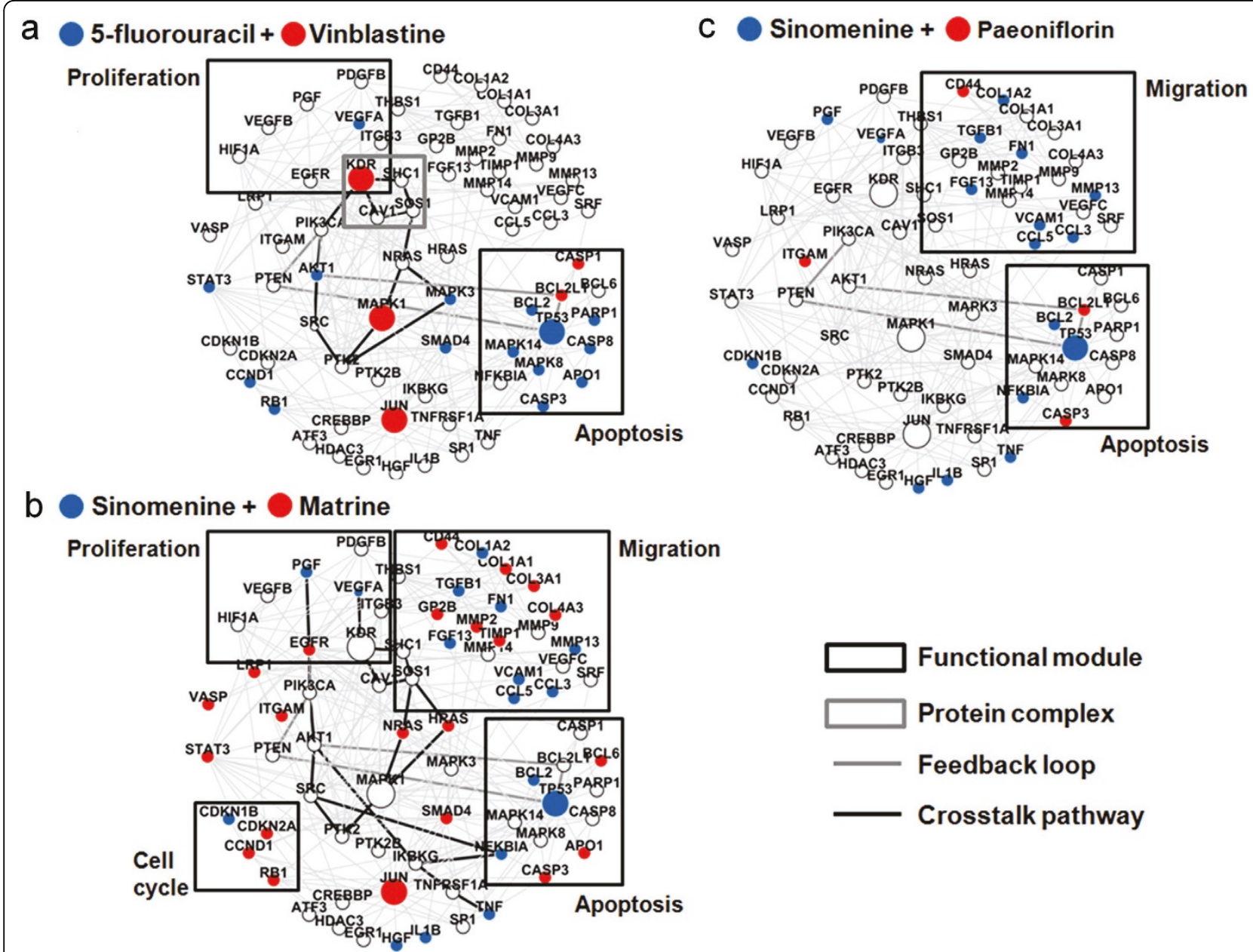

Figure 4 Features of synergistic agent combinations on the angiogenesis network target. a. 5-flourourcil and Vinblastine with known synergy. b. Sinomenine and Matrine with the high NIMS synergy score. c. Sinomenine and Paeoniflorin with the low NIMS synergy score. The nodes with red or blue colour denote responsive genes associated with two agents respectively.

against the angiogenesis network, could not only successfully recover known synergistic drug pairs (Table 1), but also rank the anti-angiogenesis synergistic agents matched with a given agent, Sinomenine (Figure 2). Interestingly, two synergistic agent pairs predicted by NIMS in the case study, Sinomenine and Matrine, and Sinomenine and Honokiol, respectively, are main constituents of TCM herbal formulae such as Qing-Luo-Yin [38] and Tou-Gu-Zhen-Feng pill. These preliminary results demonstrate the potential of NIMS as a tool for screening synergistic combinations from current drugs as well as TCM herbs or herbal formulae.

NIMS uses the agent gene and phenotype information plus network topology features. We demonstrated that NIMS is robust to the collected agent genes if the key genes are reserved (Figure $3 \mathrm{a}$ and Figure $3 \mathrm{~b}$ ). Moreover, NIMS is also relatively robust to the background network, although available networks such as the PPI network are still incomplete and biased (Figure 3c) [54].
We consider the following aspects of NIMS may contribute to such robust performances. (1) The gene set information of agents not only reflects the knowledge of agent action similarity, but also determines the meet/ min coefficient. We detected a potential correlation between the meet/min coefficient and the NIMS score. Thus the agent gene information itself ensures a relatively stable performance of NIMS against different types of networks. (2) The inherent agreement of topological features, a critical element in ranking synergistic agent pairs, is embedded in the angiogenesis, HPRD and KNC networks. On the contrary, poor performance is seen when the network topology is fundamentally altered, as in the MNC pathway network (Table 1). Note that the MNC pathway network is constructed in a different way (See Methods for details). (3) NIMS only makes use of a small fraction of the network around the network targets. Thus, it is relatively insensitive to changes of the whole background network but 


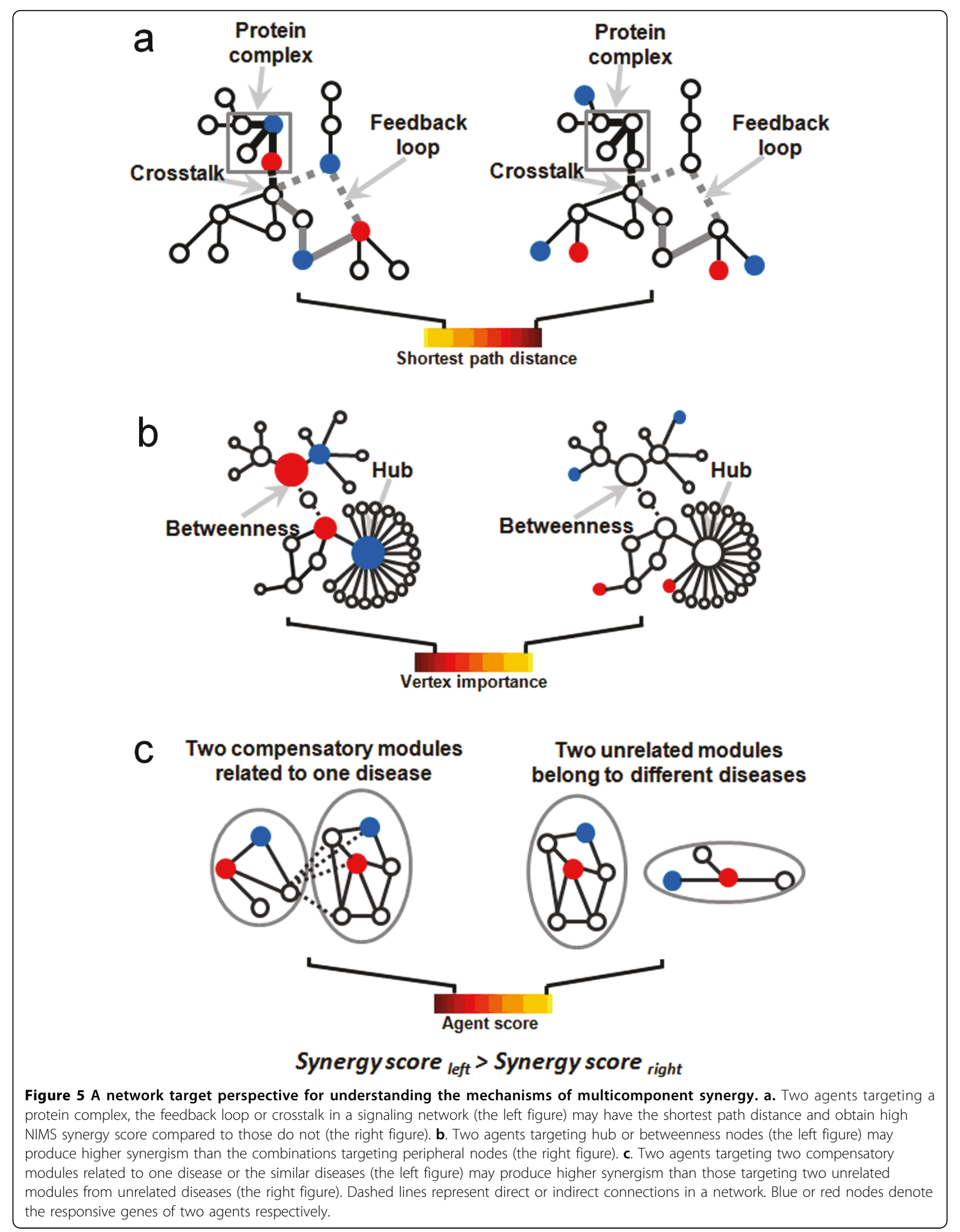


very sensitive to changes in key genes. This fact underlines the importance of the network target as a determining factor responsible for both disease mechanisms and agent actions in a network level.

We also evaluated the underlying synergy strengths produced by agent pairs from the perspective of GO functions. For 62 agents matched with Sinomenine, there is relatively lower correlation between NIMS synergy scores and GO co-annotations (Table 3). This finding is not surprising, because synergistic effects in multicomponent therapeutics could be achieved by genes that are involved in different biological processes related to a disease [1]. A disease or pathological condition is also characterized by the involvement of complex biological processes with hierarchical organization. Hence, synergistic agent pairs may not be restricted to act on the same biological functions.

Based on the above results, we further investigated the effects on the angiogenesis network target illustrated by three agent combinations with different NIMS synergy scores, namely 5-flourourcil and Vinblastine, Sinomenine and Matrine, and Sinomenine and Paeoniflorin (Figure 4), and characterized the multicomponent synergistic mechanisms from a network target perspective (Figure 5). Interestingly, we found that the network target coupling with NIMS parameters can capture the potential drug synergistic mechanisms from many aspects covering protein complexes, crosstalk and feedback loop of pathways, and stimuli-influenced molecular number [42-52], demonstrating the network target could serve as a new mode of drug target and the NIMS method is reasonable for identifying drug synergy. Such features also make NIMS compatible and upgradeable with other small-scale or large-scale network methods regarding drug action mechanisms we developed recently $[9,55][22]$.

NIMS is a vital part in our NIDA (Network targetbased Identification of Drug Action and drug synergy) system [56]. In previous studies, we demonstrate that this system can also be used to prioritize effects of candidate drugs / herbs on one or more biological processes related to given diseases [57]. To improve further the quality and performance of NIMS, there are three issues to be considered. First, the network target for a specific disease can be generated by disease-causal gene networks, disease-responsive gene networks or drug target networks. Due to the lack of understanding of complex diseases, here we only adopt the responsive gene network associated with a given disease or pathological process such as angiogenesis. It is believed that the more precise the network target is chosen, the more accurate predictions will be obtained, as suggested by the comparison results between the angiogenesis network and three global networks. We will also evaluate more useful parameters such as subgraph centrality and information centrality to calculate the node importance in both directed and undirected networks [58]. Additionally, the prediction obtained by NIMS may also be improved if we make use of more information such as the network Yin-Yang imbalance [25] or the side-effect information to refine the network target.

Second, though we only conducted the pure compounds to experimental studies, NIMS actually can be flexibly used to multiple ingredients in each herb as long as the related genes (agent genes) are available and reliable. To extend NIMS to more complicated conditions or more than two agents, we can treat mixed agents such as herb extracts and herbs as a group of compounds, and the predicted ranks of NIMS depend only on what agent genes are inputted and how accurate the agent genes are. For agent genes, the present work merely considered responsive genes associated with a limited number of TCM agents. Hopefully, NIMS can be updated when more precise information on drug targets is revealed for more agents by experiments or recent developed prediction tools such as drugCIPHER [55].

Third, as an initial effort for prioritizing synergistic agent combination in a computational framework, NIMS currently is a little bit simplified since it considers only part of the synergistic effects at the molecular level and currently does not make the distinction between the synergistic and antagonistic effects. The tissue-level synergism did not enter into our calculations. Further studies will be devoted to quantitative analysis of synergy, tissue-level synergy analysis, and pattern comparison between synergism and antagonism by integrating multilayer -omic data and spatio-temporal information. The identification of the cooperative behaviours and mechanisms of multiple agents as well as corresponding network targets will also be examined by both in vitro and in vivo experiments.

\section{Conclusions}

In summary, our work demonstrates that the network target-based methods are of importance for estimating synergistic combinations and facilitating the combinational drug development. NIMS can serve as a first-step computational approach for the high-throughput identification of multicomponent synergy and the modernization of traditional Chinese medicine. It is also a promising way to elucidate the inter-relationship between complex diseases and drug interventions through the network target paradigm.

\section{Methods}

\section{Data preparation}

To obtain the empirical multicomponent candidates, 49 TCM herbs and 12 herb-derived compounds with 
potential anti-inflammatory, anti-angiogenic or antitumor activities were selected from the 2005 Edition of Chinese Pharmacopoeia, an official compendium of drugs, covering traditional Chinese herbs, herbal formulae and western medicines.Two chemicals 5-fluorouracil and Rapamycin were also included and resulted in a total of 63 agents. Five agent pairs among them were reported synergistic action and retrieved as benchmark data for NIMS outputs. By reading more than 2,000 references regarding agent actions from both PubMed and the China National Knowledge Infrastructure (http://www.cnki.net), available agent genes and agent phenotypes were manually collected. The number of genes for each agent ranged from 10 to 108. A total of 736 non-redundant agent genes were obtained. For calculating Agent Score $(A S)$, we collected the agent phenotype similarity scores from the study of van Driel et al [31], in which the similarity score between two phenotypes is determined by the cosine of their feature vector angle, and the reliability of the score has also been tested [31].

\section{Angiogenesis network construction and three global networks}

The angiogenesis gene network was constructed by the LMMA method we developed previously [17]. By using the keyword "Angiogenesis OR Neovascularization", we retrieved 49,885 PubMed abstracts (until Feb 9, 2007), in which 2,707 genes were identified with Entrez gene ids and served as nodes of the angiogenesis network. Two genes were considered linked if they had any relationship in the PPI from HPRD (release 7) [59] or pathway interactions from KEGG [60]. We also employed three types of global networks, the PPI network and two types of global pathway networks merged from 201 KEGG human pathways, to evaluate the robustness of NIMS in terms of the background network. In KEGG, one node within a KEGG Orthology (KO) may denote a group of genes/proteins, and one gene may belong to different KOs. For example, K01090 contains 26 human genes, and the gene CDKN3 is categorized in both K01090 and K01104. Therefore, we built two distinct pathway networks: the Keep Node Content pathway network and the Merge Node Content pathway network. In the KNC network, the original node content was kept consistent, whereas in the MNC network, different KOs with one or more overlapping genes were merged into one node.

\section{NIMS robustness analysis}

By changing the parameters and then calculating the correlation between the new and original NIMS scores, we checked whether NIMS could perform robustly. All three centrality measures (Betweenness, Closeness and
PageRank) for TS and the role of $A S$ were analyzed. Then, we conducted permutation tests and measured SRCC between the permutated and original TS or AS scores for the changes of collected agent genes as well as the background networks. In this step, agent genes were removed or added randomly from the angiogenesis network, changing $10 \%$ of the genes at a time. Each iteration of adding or removing genes was repeated 100 times. For angiogenesis network, we randomly deleted edges and imported additional edges respectively at different percentages, each repeated 20 times, and measured the synergy score.

\section{NIMS synergy and GO function analysis}

To examine the association between biological functions and the NIMS predicited synergy, we used permutation tests and SRCC to evaluate whether the genes related to the synergistic agent pairs predicted by NIMS tended to have co-annotations in GO [61]. We used the UnionIntersection $(U I)$ score to analyze the GO functional similarity for genes from each agent pair. The $U I$ score was calculated by the GOstats package in Bioconductor [62], defined as $U I_{g_{1}, g_{2}}=\frac{\left|G O s_{-} g_{1} \cap G O s_{-} g_{2}\right|}{\left|G O s_{-} g_{1} \cup G O s_{-} g_{2}\right|}$, where GOs $\_g_{1}$ and GOs $\_g_{2}$ are the GO annotation term sets of

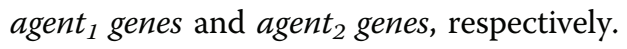

\section{Angiogenesis in vitro assay}

We employed the commonly-used Endothelial Cell Proliferation assay to verify NIMS predicted synergistic effects on angiogenesis. Human Umbilical Vein Endothelial Cells were obtained from Cascade Biologics (Portland, USA), cultured in Medium 200 (Cascade Biologics), supplemented with low serum growth supplement including $2 \%$ fetal bovine serum and a welldocumented angiogenic growth factor bFGF $(5 \mathrm{ng} / \mathrm{ml})$ stimulus. Sinomenine and the sampled partner agents were purchased from the National Institute for the Control of Pharmaceutical and Biological Products, Beijing, China. The concentration range of each agent was obtained from literature and the IC50 value (the half maximal inhibitory concentration) for each individual agent was measured. To compare the interacted agents under the same effect level, we determined the proportion of each agent pair by following the same ratio of the two agent's IC50 values. For example, if the IC50 values of agent $_{1}$ and $a g e n t_{2}$ are 10 and 100 respectively, we set the proportion of this agent pair as 1:10 in verification experiments. Each treatment was administrated after cell growth for 24 hours in a 96 -well plate. Cell proliferation was estimated using a Cell Counting Kit (CCK-8, Dojindo, Japan) after 48 hours of treatment. Each experiment was repeated three times. By using the 
Bliss independence model [5], the synergistic strength was determined by calculating: $M I I R=\max \left(I R_{s y n}-I R_{a d d}\right)$, where $I R_{s y n}$ and $I R_{a d d}$ denote inhibition rates and the Bliss additive value of an agent pair at a certain dose/ ratio.

\section{Acknowledgements}

This work is supported by the National Natural Science Foundation of China (Nos. 30873464, 60934004 and 61021063), 2010THZ0 and NCET-07-0486. This article has been published as part of BMC Systems Biology Volume 5 Supplement 1, 2011: Selected articles from the 4th International Conference on Computational Systems Biology (ISB 2010). The full contents of the supplement are available online at http://www.biomedcentral.com/17520509/5? issue $=$ S1

\section{Authors' contributions}

$S L$ conceived and designed the experiments, analyzed the data and wrote manuscript. BZ participated in cell experiments, data analysis and writing manuscript. NZ participated in computational works.

\section{Competing interests}

The authors declare that they have no competing interests.

Published: 20 June 2011

\section{References}

1. Zimmermann GR, Lehar J, Keith CT: Multi-target therapeutics: when the whole is greater than the sum of the parts. Drug Discov Today 2007, 12:34-42.

2. Keith $C T$, Borisy AA, Stockwell BR: Multicomponent therapeutics for networked systems. Nat Rev Drug Discov 2005, 4:71-78.

3. Dancey JE, Chen HX: Strategies for optimizing combinations of molecularly targeted anticancer agents. Nat Rev Drug Discov 2006, 5:649-659.

4. Berenbaum MC: Criteria for analyzing interactions between biologically active agents. Adv Cancer Res 1981, 35:269-335.

5. Bliss Cl: The calculation of microbial assays. Bacteriol Rev 1956, 20:243-258.

6. Loewe $\mathrm{S}$ : The problem of synergism and antagonism of combined drugs. Arzneimittelforschung 1953, 3:285-290.

7. Chou TC, Talalay P: Analysis of combined drug effects: a new look at a very old problem. Trends Pharmacol Sci 1983, 4:450-454.

8. Borisy AA, Elliott PJ, Hurst NW, Lee MS, Lehar J, Price ER, Serbedzija G, Zimmermann GR, Foley MA, Stockwell BR, Keith CT: Systematic discovery of multicomponent therapeutics. Proc Natl Acad Sci U S A 2003, 100:7977-7982.

9. Yan H, Zhang B, Li S, Zhao Q: A formal model for analyzing drug combination effects and its application in TNF-a-induced NFKB pathway. BMC Syst Biol 2010, 4:50.

10. Fitzgerald JB, Schoeberl B, Nielsen UB, Sorger PK: Systems biology and combination therapy in the quest for clinical efficacy. Nat Chem Biol 2006, 2:458-466.

11. Araujo RP, Liotta LA, Petricoin EF: Proteins, drug targets and the mechanisms they control: the simple truth about complex networks. Nat Rev Drug Discov 2007, 6:871-880.

12. Lu LJ, Sboner A, Huang YPJ, Lu HX, Gianoulis TA, Yip KY, Kim PM, Montelione GT, Gerstein MB: Comparing classical pathways and modern networks: towards the development of an edge ontology. Trends Biochem Sci 2007, 32:320-331

13. Huang $Y, L i$ S: Detection of characteristic sub pathway network for angiogenesis based on the comprehensive pathway network. BMC Bioinformatics 2010, 11(Suppl 1):S32.

14. Csermely $P$, Agoston $V$, Pongor S: The efficiency of multi-target drugs: the network approach might help drug design. Trends Pharmacol Sci 2005, 26:178-182.

15. Wu X, Jiang R, Zhang MQ, Li S: Network-based global inference of human disease genes. Mol Syst Biol 2008, 4:189.

16. Kitano $\mathrm{H}$ : Cancer as a robust system: implications for anticancer therapy. Nat Rev Cancer 2004, 4:227-235.
17. Li S, Wu LJ, Zhang ZQ: Constructing biological networks through combined literature mining and microarray analysis: a LMMA approach. Bioinformatics 2006, 22:2143-2150.

18. Schadt EE: Molecular networks as sensors and drivers of common human diseases. Nature 2009, 461:218-223.

19. Hopkins AL: Network pharmacology: the next paradigm in drug discovery. Nat Chem Biol 2008, 4:682-690.

20. Fan TP, Yeh JC, Leung KW, Yue PYK, Wong RNS: Angiogenesis: from plants to blood vessels. Trends Pharmacol Sci 2006, 27:297-309.

21. Williamson EM: Synergy and other interactions in phytomedicines. Phytomedicine 2001, 8:401-409.

22. Li S, Zhang B, Jiang D, Wei YY, Zhang NB: Herb network construction and co-module analysis for uncovering the combination rule of traditional Chinese herbal formulae. BMC Bioinformatics 2010, 11(Suppl 11):S6.

23. Wang L, Zhou GB, Liu P, Song JH, Liang Y, Yan XJ, Xu F, Wang BS, Mao JH, Shen ZX, et al: Dissection of mechanisms of Chinese medicinal formula Realgar-Indigo naturalis as an effective treatment for promyelocytic leukemia. Proc Natl Acad Sci U S A 2008, 105:4826-4831.

24. Schmidt BM, Ribnicky DM, Lipsky PE, Raskin I: Revisiting the ancient concept of botanical therapeutics. Nat Chem Biol 2007, 3:360-366.

25. Li S, Zhang ZQ, Wu L, Zhang XG, Li YD, Wang YY: Understanding ZHENG in traditional Chinese medicine in the context of neuro-endocrineimmune network. IET Syst Biol 2007, 1:51-60

26. Arrell DK, Terzic A: Network systems biology for drug discovery. Clin Pharmacol Ther 2010, 88:120-125.

27. Hwang WC, Zhang A, Ramanathan M: Identification of information flowmodulating drug targets: a novel bridging paradigm for drug discovery. Clin Pharmaco Ther 2008, 84:563-572.

28. Nacher JC, Schwartz JM: A global view of drug-therapy interactions. BMC Pharmacol 2008, 8:5-14.

29. Korcsmáros T, Szalay MS, Böde C, Kovács IA, Csermely P: How to design multi-target drugs : target search options in cellular networks. Expert Opin Drug Discov 2007, 2:1-10.

30. Page L, Brin S, Motwani R, Winograd T: The Pagerank Citation Ranking: Bringing Order to the Web. Technical report Stanford Digital Libraries; 1998.

31. van Driel MA, Bruggeman J, Vriend G, Brunner HG, Leunissen JAM: A textmining analysis of the human phenome. Eur J Hum Genet 2006, 14:535-542.

32. Folkman J: Angiogenesis in cancer, vascular, rheumatoid and other disease. Nat Med 1995, 1:27-31

33. Vergeylen A, Kloppel G: Vinblastine and 5-fluorouracil sensitivity of xenografts of four pancreatic ductal adenocarcinomas: is there a correlation with histological and cytological tumour differentiation? Virchows Arch 1995, 427:317-322.

34. Seeliger H, Guba M, Koehl GE, Doenecke A, Steinbauer M, Bruns CJ, Wagner C, Frank E, Jauch KW, Geissler EK: Blockage of 2-deoxy-D-riboseinduced angiogenesis with rapamycin counteracts a thymidine phosphorylase-based escape mechanism available for colon cancer under 5-fluorouracil therapy. Clin Cancer Res 2004, 10:1843-1852.

35. El-Galley R, Keane TE, Sun C: Camptothecin analogues and vinblastine in the treatment of renal cell carcinoma: an in vivo study using a human orthotopic renal cancer xenograft. Urol Oncol 2003, 21:49-57.

36. Papazisis KT, Kalemi TG, Zambouli D, Geromichalos GD, Lambropoulos AF, Kotsis A, Boutis LL, Kortsaris AH: Synergistic effects of protein tyrosine kinase inhibitor genistein with camptothecins against three cell lines in vitro. Cancer Lett 2006, 233:255-264.

37. Puli S, Jain A, Lai JC, Bhushan A: Effect of combination treatment of rapamycin and isoflavones on mTOR pathway in human glioblastoma (U87) cells. Neurochem Res 2010, 35:986-993.

38. Li S, Lu A, Wang Y, Li Y: Suppressive effects of a Chinese herbal medicine Qing-Luo-Yin extract on the angiogenesis of collagen induced arthritis in rats. Am J Chin Med 2003, 31:713-720.

39. Menéndez JA, del Mar Barbacid M, Montero S, Sevilla E, Escrich E Solanas M, Cortés-Funes H, Colomer R: Effects of gamma-linolenic acid and oleic acid on paclitaxel cytotoxicity in human breast cancer cells. Eur J Cancer 2001, 37:402-413.

40. Albert R, Barabási AL: Statistical mechanics of complex networks. Reviews of Modern Physics 2002, 74:47-97.

41. Goldberg DS, Roth FP: Assessing experimentally derived interactions in a small world. Proc Natl Acad Sci U S A 2003, 100:4372-4376. 
42. Jansen G, Lee AY, Epp E, Fredette A, Surprenant J, Harcus D, Scott M, Tan E, Nishimura T, Whiteway $M$, et al: Chemogenomic profiling predicts antifungal synergies. Mol Syst Biol 2009, 5:338-350.

43. Li Y, Agarwal P, Rajagopalan D: A global pathway crosstalk network. Bioinformatics 2008, 24:1442-1447.

44. López-Otín C, Hunter T: The regulatory crosstalk between kinases and proteases in cancer. Nat Rev Cancer 2010, 10:278-292.

45. Chakraborty AK, Liang K, DiGiovanna MP: Co-targeting insulin-like growth factor I receptor and HER2: dramatic effects of HER2 inhibitors on nonoverexpressing breast cancer. Cancer Res 2008, 68:1538-1545.

46. Fraser ID, Germain RN: Navigating the network: signaling cross-talk in hematopoietic cells. Nat Immunol 2009, 10:327-331.

47. Jia J, Zhu F, Ma X, Cao Z, Li Y, Chen YZ: Mechanisms of drug combinations: interaction and network perspectives. Nat Rev Drug Discov 2009, 8:111-128.

48. Fitzgerald JB, Schoeberl B, Nielsen UB, Sorger PK: Systems biology and combination therapy in the quest for clinical efficacy. Nat Chem Biol 2006, 2:458-466.

49. Lehár J, Krueger A, Zimmermann G, Borisy A: High-order combination effects and biological robustness. Mol Syst Biol 2008, 4:215-220.

50. Fliri AF, Loging WT, Volkmann RA: Drug effects viewed from a signal transduction network perspective. J Med Chem 2009, 52:8038-8046.

51. Barrera NP, Morales B, Torres S, Villalón M: Principles: mechanisms and modeling of synergism in cellular responses. Trends Pharmacol Sci 2005, 26:526-532.

52. Cline El, Bicciato S, DiBello C, Lingen MW: Prediction of in vivo synergistic activity of antiangiogenic compounds by gene expression profiling. Cancer Res 2002, 62:7143-7148.

53. Hawk E, Viner JL: Statins and cancer-beyond the "one drug, one disease" model. N Engl J Med 2005, 352:2238-2239.

54. Hakes L, Pinney JW, Robertson DL, Lovell SC: Protein-protein interaction networks and biology-what's the connection? Nat Biotech 2008, 26:69-72.

55. Zhao S, Li S: Network-based relating pharmacological and genomic spaces for drug target identification. PLOS ONE 2010, 5:e11764.

56. Li S, Zhang NB: NIDA: Methods of network target-based identification of drug action and drug synergy. 2009, Invention Patent. PCT/CN/074377.

57. Li LS, Zhang NB, Zhang B, Li S: Ranking effects of candidate drugs on biological process by integrating network analysis and Gene Ontology. Chinese Sci Bull 2010, 55:2974-2980.

58. Wang J, Zhang S, Wang Y, Chen L, Zhang XS: Disease-aging network reveals significant roles of aging genes in connecting genetic diseases. PLoS Comput Biol 2009, 5:e1000521.

59. Peri $S$, Navarro JD, Amanchy R, Kristiansen TZ, Jonnalagadda CK, Surendranath V, Niranjan V, Muthusamy B, Gandhi TK, Gronborg M, et al: Development of human protein reference database as an initial platform for approaching systems biology in humans. Genome Res 2003, 13:2363-2371.

60. Kanehisa M, Araki M, Goto S, Hattori M, Hirakawa M, Itoh M, Katayama T, Kawashima S, Okuda S, Tokimatsu T, Yamanishi Y: KEGG for linking genomes to life and the environment. Nucleic Acids Res 2008, 36: D480-484.

61. Ashburner M, Ball CA, Blake JA, Botstein D, Butler H, Cherry JM, Davis AP, Dolinski K, Dwight SS, Eppig JT, et al: Gene ontology: tool for the unification of biology. The Gene Ontology Consortium. Nat Genet 2000, 25:25-29.

62. Gentleman RC, Carey VJ, Bates DM, Bolstad B, Dettling M, Dudoit S, Ellis B, Gautier L, Ge Y, Gentry J, et al: Bioconductor: open software development for computational biology and bioinformatics. Genome Biol 2004, 5:R80.

doi:10.1186/1752-0509-5-S1-S10

Cite this article as: Li et al:: Network target for screening synergistic drug combinations with application to traditional Chinese medicine. BMC Systems Biology 2011 5(Suppl 1):S10.

\section{Submit your next manuscript to BioMed Central and take full advantage of:}

- Convenient online submission

- Thorough peer review

- No space constraints or color figure charges

- Immediate publication on acceptance

- Inclusion in PubMed, CAS, Scopus and Google Scholar

- Research which is freely available for redistribution

Submit your manuscript at www.biomedcentral.com/submit
C Biomed Central 